\title{
Modelo para un tratamiento de errores de transferencia en la escritura en ELE producida en comunidades virtuales ${ }^{1}$
}

\author{
Model for Treating Transfer Errors in SFL Writing \\ Produced in Virtual Communities
}

\author{
Modelo para um tratamento de erros de transferência na \\ escrita em ELE produzida em comunidades virtuais
}

Jessica Elejalde Gómez ${ }^{2}$ Anita Ferreira Cabrera ${ }^{3}$

\section{Resumen}

El tratamiento de errores, en general, busca corregir aquellos relacionados con las normas del sistema lingüístico en la lengua objeto de estudio. Sin embargo, son escasas las propuestas que intervienen en los errores según su fuente u origen, como en el caso de la influencia directa o indirecta de la lengua materna o aquellos que son parte del desarrollo de la interlengua. En este contexto, se expone la propuesta de un modelo para tratar errores de transferencia de la lengua materna a partir de la sensibilización de este tipo error en la producción escrita por parte del aprendiente. Dicho modelo ha surgido de la observación en estudio de la interacción que ocurre en comunidades de aprendizaje virtual, donde los aprendientes practican la escritura de forma espontánea y libre y, otros pares de aprendientes retroalimentan la escritura en relación con los errores identificados basados en su propia intuición 0 conocimiento lingüístico que posean. Dicha observación se realizó durante un determinado tiempo,en el marco de la investigación realizada por Elejalde y Ferreira (2016) donde los errores identificados fueron en su mayoría provenientes de la influencia directa de la lengua materna.Este modelo favorece a docentes y estudiantes de lenguas en el proceso de identificación de errores de transferencia. De esta manera se contribuye a la sensibilización y concienciación por parte del estudiante de dichos errores y su adecuada corrección en diversas situaciones comunicativas.

Palabras clave

errores de transferencia; tratamiento de errores; análisis de errores; corpus de aprendientes

Abstract

Error treatment, in general, aims to correct those errors related to the rules of the linguistic system in the target language. However, there are few proposals to deal with errors according to their source or origin, as in the case of direct or indirect influence of the mother tongue or errors appearing during the development of the interlanguage. In this context, we propose a model for treating mother tongue transfer errors, based on making the learner aware of this type of errors in the written production. This model emerged from the observational study of interaction in virtual learning communities, where learners practice writing spontaneously and freely, and other learners give feedback to the writing regarding the errors identified based on their own intuition or their linguistic knowledge. This observation took place over a certain amount of time in the context of the research conducted by Elejalde and Ferreira (2016), in which most of the identified errors came from the direct influence of the mother tongue. This model helps language teachers and students in the process of identifying transfer errors, thus contributing to making the students aware of said errors and their proper correction in different communicative situations.

\section{Keywords}

transfer errors; error treatment; error analysis; learner's corpus

1 Este modelo pertenece al desarrollo de la tesis de investigación de la autora Jessica Elejalde y la profesora guía Anita Ferreira. El estudio sobre el cual se basa este modelo puede ser consultado en el artículo publicado por Elejalde y Ferreira (2016). Asimismo, la tesis fue financiada por la beca Conicyt Doctorado Nacional 2013 y por fondos de investigación del modelo de análisis, procesamiento y corrección de errores del Proyecto Fondecyt No.1140651: "El feedback correctivo escrito directo e indirecto en la adquisición y aprendizaje del español como lengua extranjera" de Anita Ferreira.

2 Universidad de Concepción. Correo electrónico: jelejalde@udec.cl

3 Universidad de Concepción. Correo electrónico: aferreir@udec.cl 


\section{Resumo}

0 tratamento de erros, em geral, procura corrigir aqueles relacionados com as normas do sistema linguístico na língua objeto de estudo. Contudo, são poucas as propostas relacionadas com a intervenção nos erros segundo sua fonte e origem, como no caso da influência direta ou indireta da língua materna ou aqueles que são parte do desenvolvimento da interlíngua. Neste contexto, apresenta-se a proposta de um modelo para o tratamento dos erros de transferência da língua materna a partir da sensibilização desse tipo de erro na produção de textos por parte do aprendente. Esse modelo surgiu da observação no estudo da interação que acontece em comunidades de aprendizagem virtual, onde os aprendentes praticam a escrita de forma livre e espontânea, recebendo retroalimentação de outros aprendentes, na que identificam erros produzidos na escrita, com base no seu conhecimento linguístico ou intuição. Essa observação realizou-se durante a pesquisa desenvolvida por Elejade e Ferreira (2016), onde os erros identificados foram principalmente causados pela influência direta da língua materna. Este modelo beneficia estudantes e professores de línguas no processo de identificação de erros de transferência, contribuindo, dessa forma, para a sensibilização e conscientização por parte do estudante desses erros e sua correção em diversas situações comunicativas.

\section{Palavras-chave}

erros de transferência; tratamento de erros; análise de erros; corpus de aprendentes

\section{Artículo recibido el 24 de agosto de 2017 y aprobado el 10 de enero de 2018}

\section{Introducción}

El tratamiento del error en cualquier proceso de aprendizaje está supeditado a diferentes variables: el tipo de error, el contexto de aprendizaje, el estilo y las diferencias individuales, la necesidad, los fenómenos subyacentes a la conformación de la interlengua, el conocimiento del error y el interés del estudiante por repararlo. Dentro de estas variables, una de la más importante se refiere al conocimiento que tenga el estudiante de sus propias dificultades en el aprendizaje y cómo estas se manifiestan en su producción oral y escrita. Asimismo, el contexto de aprendizaje desempeña un papel relevante dada las condiciones que propicia para una práctica escritural, como en el caso de las comunidades virtuales, espontánea y disponible para ser vista y validada por otros.

Con respecto a los errores, los de tipo lingüístico han sido ampliamente discutidos en materia de investigación y reparación en clase presencial, sin embargo, los aprendices de lenguas, en la mayoría de veces, no son conscientes de cómo su lengua materna es responsable de muchos de estos. Es decir, los errores gramaticales, léxicos o discursivos, en gran parte son producto de la influencia directa o indirecta de la lengua materna, afectando la comunicación en la lengua objeto de estudio (LO) acorde con el nivel de dominio que tenga de esta. Dicho desconocimiento podría ocurrir, en primer lugar, porque el aprendiente desconoce la estrategia de aprendizaje que permite solucionar problemas de comunicación a partir de su sistema lingüístico materno.
Esta estrategia, conocida en el ámbito de la adquisición de segundas lenguas (ASL) como la transferencia interlingual o interferencia lingüística, puede producir efectos positivos o negativos dependiendo de varios factores asociados a la Lo y la lengua materna (LM). En segundo lugar, las transferencias originadas en la producción del aprendiente muchas veces pueden $o$ no ser identificadas por parte del estudiante debido al desconocimiento tanto de su propia lengua materna (en cuestión gramatical y morfosintáctica) como la desinformación respecto del sistema lingüístico de la Lo. Pero, ¿de qué manera se podría intervenir este tipo de errores de transferencia considerando un ambiente de aprendizaje mediatizado por la tecnología? Y ¿cómo esta propuesta podría extrapolarse al contexto de clases presenciales?

Hay varias metodologías para intervenir y reparar los errores lingüísticos y léxicos (Alexopolou, 2005; Ferreira y Kotz, 2010; Ferreira, Salcedo, Kotz y Barrientos 2012; Vázquez, 2009); no obstante, abordar las clasificaciones en errores de transferencia se vuelve complejo, dada la dificultad, por parte de quien los corrige, para identificar la fuente del error. La identificación de errores de transferencia interlingual implica conocer el sistema de la LM en contraste con la LO. En este sentido, a pesar de presentarse aulas o comunidades virtuales de clase multilingües con diversas culturas, los profesores de lenguas no siempre cuentan con el conocimiento de la LM de los estudiantes, lo que dificulta el reconocimiento de errores provenientes de la LM. Aquellos 
estudiantes que tengan lenguas similares o lenguas en común conocidas por el profesor, podrán reconocer con mayor precisión los errores, sin embargo, debe haber una preparación por parte del profesor de ELE en este tipo de errores de transferencia, cuya identificación y posterior tratamiento asegurará el mejoramiento continuo de la conformación de la interlengua.

En ese sentido, en este artículo se presenta un modelo de tratamiento para errores de transferencia, producto de un estudio de análisis de errores de estudiantes que escribieron en ELE en ambientes de aprendizaje mediatizado por la tecnología.

\section{Fundamento teórico}

Acorde con la propuesta de un modelo de tratamiento de errores, los sustentos teóricos se centran en modelos anteriormente propuestos y el tratamiento de errores en ambientes de aprendizaje presencial y en línea.

\section{La escritura en las comunidades virtuales y la influencia de la lengua materna}

Las comunidades de aprendizaje de lenguas en línea están basadas en la comunicación e intercambio de información mediada por el computador (communication mediated computer, $\mathrm{CMC}$ ). La comunicación que surge allí se produce en la escritura, la lectura y oralidad vía Networked, donde se practican las habilidades lingüísticas para aprender una segunda lengua (Warschauer, 2001). Entre estas posibilidades de uso, los aprendientes de las comunidades obtienen beneficios del intercambio con otros hablantes nativos, dado el interés por el aprendizaje de una lengua extranjera común o la del aprendiente (Lamy y Hampel, 2007).

A través de la comunicación vía computacional, estas comunidades de lenguas se han convertido en un espacio de interacción casi exclusivamente escrito, basado en el intercambio vía chat (sincrónico) o en la publicación de textos escritos resultados de la práctica en una lengua extranjera (asincrónico).
La escritura en estos sitios es libre, espontánea y el uso de la lengua meta es real en contextos de comunicación auténtica. De esta forma, se convierte en un proceso colaborativo en el que se propicia la interacción, la negociación del significado y la práctica de la lengua extranjera en diversas situaciones comunicativas (Long, 1996; Lamy y Hampel, 2007). En este aspecto, cuando el aprendiente se enfrenta al uso de la L2 en un ambiente natural, recurre a diferentes estrategias de comunicación o de aprendizaje, entre las cuales la lengua materna (LM) es la primera opción. El uso de la LM cobra importancia al suplir vacíos de información lingüística, y otras estrategias como la sobregeneralización o neutralización permiten replicar reglas ya aprendidas en la L2.

Asimismo, los aprendientes en este ambiente pueden controlar la ansiedad o el nerviosismo causados por la participación presencial en el aula de clases (Lamy y Hampel, 2007). Por ello, la producción escrita digital propicia un nuevo procedimiento de escritura, donde su relevancia y análisis es similar a la de cualquier otro tipo de texto generado en otro medio físico (Cassany, 2006).

\section{Modelos de tratamiento de errores y propuestas didácticas en ELE}

Tratar errores de cualquier tipo requiere de técnicas y estrategias que permitan al aprendiente profundizar en la identificación y reflexión de los errores señalados por el profesor. De esta forma, se debe orientar la intervención correctiva hacia una sensibilización y reparación del aprendiente donde no le reste importancia al error y no se desmotive con la corrección, sino que se produzca la reflexión sobre las problemáticas observadas en su interlengua y, como consecuencia, la corrección de estos (Alexopoulou, 2005). En esta línea, los estudios donde la intervención correctiva está centrada en la reflexión del aprendiente, tiene como finalidad tres aspectos relevantes:

- La sensibilización del aprendiente ante ciertos fenómenos interlingüísticos o intralingüísticos producidos durante la conformación de la interlengua. 
- Apoyo para el reconocimiento e identificación de los errores y la autocorrección.

- Fomentar su interés por el monitoreo, seguimiento y clarificación en cuanto a los errores que surgen durante su proceso de aprendizaje.

Para lograr la operacionalización de estos aspectos en el aprendizaje es necesario estimular la autocorrección y fomentar los espacios de participación en los que el aprendiente regule su producción. Esta postura y actitud por parte del aprendiente implica una dinámica distinta en la clase o ambiente de aprendizaje semipresencial, donde supone centrar al aprendiente como protagonista de su corrección y reparación del error.

En cuanto a la investigación en modelos de corrección y tratamiento de errores, cabe destacar la propuesta de Alexopoulou (2005), quien enfoca la reparación en la actitud del alumno siguiendo unas pautas relevantes a la hora de corregir. Estas asumen al alumno como el protagonista ante la corrección positiva. Para eso, se toman en cuenta los aspectos afectivos y sus implicaciones, cuyo efecto permite diferenciar cómo se siente el aprendiente ante la corrección. En este aspecto, la autora alude a dos elementos afectivos que pueden tener una repercusión positiva o negativa, y como consecuencia, requiere de la atención del profesor, estos son: la ansiedad y la motivación. Respecto a la primera, es necesario eliminar los factores que la fomentan en el aprendiente y lo bloquean, por ejemplo, evitar la exposición innecesaria delante de otros compañeros, o en el caso del ambiente mediatizado por la tecnología, convendría exponer los errores de acuerdo con el nivel y los errores focalizados, y por último, evitar la frustración y el temor que siente el aprendiente frente al error.

El segundo elemento, la motivación, se refiere principalmente a la repercusión que puede tener la retroalimentación, por lo que el profesor deberá considerar en la intervención correctiva efectiva lo siguiente (Alexopoulou, 2005):

- Propiciar la reflexión y la autocorrección en el aprendiente, promoviendo la capacidad del monitoreo.
- Promover la autonomía y la corresponsabilidad en el aprendizaje.

- Fomentar la corrección constructiva.

- Valorar la producción acorde con los aciertos positivos y no solo lo erróneo.

La importancia de este modelo radica en el papel centrado en el aprendiente a través de la sensibilización y la afectividad (la motivación y la ansiedad) para lograr una reparación del error. Los modelos tradicionales se centran en el error relegando el papel que tiene el alumno en su proceso de reparación (Alexopolou, 2005; Maldonado, 2013).

Con respecto a otros modelos de tratamiento centrados en el punto de vista del alumno, la propuesta didáctica de Santos Gargallo (1994) considera una serie de técnicas para la corrección de errores en la expresión escrita considerando los siguientes aspectos basados en el proceso de identificación y reflexión de la escritura en el carácter gramatical o pragmático que es relevante destacar:

- Elaboración de listas de frases incorrectas extraídas de la escritura, ante la cual el estudiante reflexione sus errores para valorar la gravedad del error.

- Comparar la producción errónea con la producción correcta, considerando la explicación gramatical incumplida, de manera que el estudiante sea consciente de sus propios errores.

- Corregir los escritos señalando en el margen la causa del error y hacer que sea el estudiante quien corrija las producciones apoyado en las gramáticas y diccionarios. Estas correcciones deben ser monitoreadas y revisadas por el profesor.

- Solicitar la reescritura del texto con las correcciones pertinentes o escribir un texto nuevo usando algunas de las estructuras del ejercicio anterior.

Santos Gargallo (1994) expresa que el tratamiento de errores y su reflexión ayudan a mejorar el conocimiento gramatical, pragmático y cultural, hecho 
que tiene un efecto positivo y directo en la capacidad para comunicarse forma efectiva en la escritura.

\section{El tratamiento de errores en ambientes \\ de aprendizaje en el aula versus \\ comunidades de aprendizaje virtual}

En el tratamiento de los errores en los diferentes espacios de aprendizaje se observan marcadas diferencias a la hora de corregir. Si bien las clases presenciales tienen la ventaja de contar con la presencia física del profesor, el ambiente generado en las comunidades virtuales también cuenta con tutores o pares de aprendices que se enfocan en la corrección de los errores (corrección en el cuaderno de notas o en los escritos publicados por los aprendientes de cada comunidad) de forma intuitiva. En estos últimos se tiene la posibilidad de una corrección directa de los pares quienes son hablantes nativos de la LO del aprendiz. En esa medida, los intercambios originados en este espacio permiten un acercamiento mayor al reconocimiento de errores de transferencia y su posible intervención.

En espacios generados como Italki.com, la comunidad participante integra la metodología de corrección basada en el conocimiento lingüístico que tenga cada aprendiente. En ese sentido, las correcciones en cada texto escrito creado en este espacio son intuitivas y obedecen a lo que cada miembro interpreta. Ahora bien, el sitio ofrece artículos destinados al mejoramiento del aprendizaje, claves para lograr una buena conversación y temas generales relacionados con el éxito de la adquisición de la lengua.

En el aula, los profesores pueden utilizar estrategias de retroalimentación correctiva basadas en diferentes tipos, como las clarificaciones, las preguntas indirectas o con un grado más de especificación, como las claves metalingüísticas, las reformulaciones entre otras; cualquiera de estas estrategias permite corregir al aprendiz acorde con un criterio de errores previamente identificados. Sin embargo, cabe mencionar y destacar que los errores de transferencia, debido a la complejidad que implica el análisis de los errores entre la lengua materna, la lengua objeto de estudio y otras lenguas aprendidas, son complejos de abordar por la diversidad que en sí representan. Como se mencionaba anteriormente, los errores de transferencia muchas veces deben ser analizados de forma contrastiva para identificar si hay interferencia de la LM hacia la Lo(errores interlingüísticos) o si es un error propio de la intralengua (errores intralingüísticos).

\section{Modelo para el tratamiento de errores producto de la transferencia de la lengua materna}

En un ambiente mediatizado por la tecnología, como las comunidades virtuales, intervenir y tratar errores se ha convertido en una herramienta importante a la hora de aprender idiomas en estos sitios. La mayoría de profesores, tutores y miembros de esa comunidad corrigen los textos escritos producidos espontáneamente para contribuir al mejoramiento de la competencia del estudiante que practica su LO; no obstante, la corrección está sujeta a la interpretación y subjetividad de cada persona que haya intervenido en la producción escrita. Aquí, el tratamiento de errores se vuelve deliberado y subjetivo, donde muchas veces la corrección puede ser un acierto o un conjunto de estimaciones que confunden el desarrollo y mejoramiento de la competencia lingüística en la LO. De igual modo, ese abordaje de las problemáticas en el uso de la lengua se orienta hacia la corrección de todos los errores, lo que podría provocar en el aprendiente estrés o desmotivación por el número de errores que debe reparar.

Un tratamiento efectivo para los errores, considerando este ambiente de aprendizaje, debe partir de la identificación, clasificación y descripción de errores, con el objeto de sensibilizar al estudiante en cuanto a los procesos que se conforman en su interlengua. Las intervenciones de los miembros de estas comunidades, si bien llevan a un punto de observación al aprendiente, sus correcciones solo se limitan a señalar los errores y explicaciones de por qué no se puede aplicar una determinada regla en la Lo. En este aspecto, proponer una metodología para corregir errores provenientes de la LM permitirá 
que la práctica de la escritura en estos sitios mejore sustantivamente en materia de errores de transferencia, y la intervención de los profesores o tutores será más pertinente y focalizada en aspectos que puede dilucidar con mayor acierto, como los errores de transferencia. Para ello, en el tratamiento es relevante determinar el tipo de error a tratar y la importancia de este para contribuir en el mejoramiento de la competencia lingüística y comunicativa en la Lo. Esta premisa, delimitada por los estudios en corrección y feedback correctivo (Ferreira, 2006; Ferreira y Lafleur, 2015; Ferreira, 2017), corresponde a la focalización que debe tenerse en cuenta al momento de tratar errores, dado que el aprendizaje debe enfocarse en los errores acorde con los niveles y estadios en los que se encuentre el aprendiente. Tratar todos los errores no solo podría causar desmotivación y estrés en el estudiante, dadas las múltiples correcciones, sino que podría manifestarse una aversión por la corrección no orientada al interés principal del objetivo de aprendizaje. Es decir, la focalización en cierto tipo de errores facilitará el aprendizaje y permitirá sensibilizar al estudiante de cada estado y etapa de corrección en diferentes aspectos de la Lo.

Para el caso que señala este modelo, los errores de transferencia han sido estudiados ampliamente en las últimas décadas para determinar las causas de su origen y formas de procesamiento en el cerebro. Sin embargo, han sido abordados escasamente para el tratamiento de la influencia de la LM hacia la Lo en el mejoramiento de la competencia lingüística de los aprendientes (Alexopoulou, 2006, 2011; Contreras y Ferreira, 2013). Las razones de esto podrían explicarse desde la complejidad que suscita interpretar el origen del error y su contraste con la LM para delimitar semejanzas o diferencias que interfieran en la interlengua. Otra razón ha sido la focalización que ha tenido la identificación de errores en el nivel lingüístico sin prestar atención en los posibles correlatos existentes entre la LM y la LO $y$, como consecuencia, su influencia en la producción escrita. Adicionalmente, se requiere del conocimiento lingüístico de las estructuras de la lengua materna en un nivel operativo categorial y léxico orientado en las colocaciones y estructuraciones semánticas, puesto que estas estructuras en la mayoría de veces no son equivalentes entre las lenguas, y esto podría llevar a una selección errónea tanto de las categorías gramaticales como del significado de las palabras. Por consiguiente, el investigador o profesor interesado en aplicar un tratamiento de este tipo de error, deberá considerar estos aspectos metodológicos y la fundamentación teórica gramatical y lingüística que represente la problemática de las lenguas en estudio.

En esta línea de consecución de etapas, se presenta un modelo de tratamiento del error basado en los procedimientos del análisis de errores $(\mathrm{AE})$ y el tratamiento de errores en ASL. Este modelo se propone para implementarlo en contextos mediatizados por la tecnología (modelos de enseñanza blendedlearning o e-learning con monitoreo del profesor o tutor) e incluso en ambientes de aprendizaje presencial con el uso del computador (figura 1).

El modelo de tratamiento presentado en la figura 1 se divide en 5 etapas: 1) identificación de los errores de transferencia basada en los criterios del análisis de errores; 2) caracterización de los errores (descripción detallada de la presencia del error según las características de la LM y la LO); 3) la sensibilización (acercamiento) del aprendiente sobre los errores de transferencia (entregar estrategias de reconocimiento de errores producto de la LM a los aprendices de ELE; 4) sensibilización dirigida y centrada en el contraste LM-LO, y 5) monitoreo y seguimiento de los errores de transferencia.

En la etapa de identificación de los errores, debe priorizarse aquellos que respondan a una categorización interlingual, con el fin de diferenciar los que son producto de la influencia de la LM. En este primer acercamiento se sugiere, desde el punto de vista del profesor, contar con herramientas de gramática contrastiva entre ambas lenguas o gramática como lengua extranjera para asegurar que la identificación tenga un análisis basado en la LM. Sobre estas consideraciones, se procede con la siguiente etapa, la caracterización del error, la cual corresponde a la explicación de este, su fuente y aparición en la producción escrita. Esto con la finalidad 


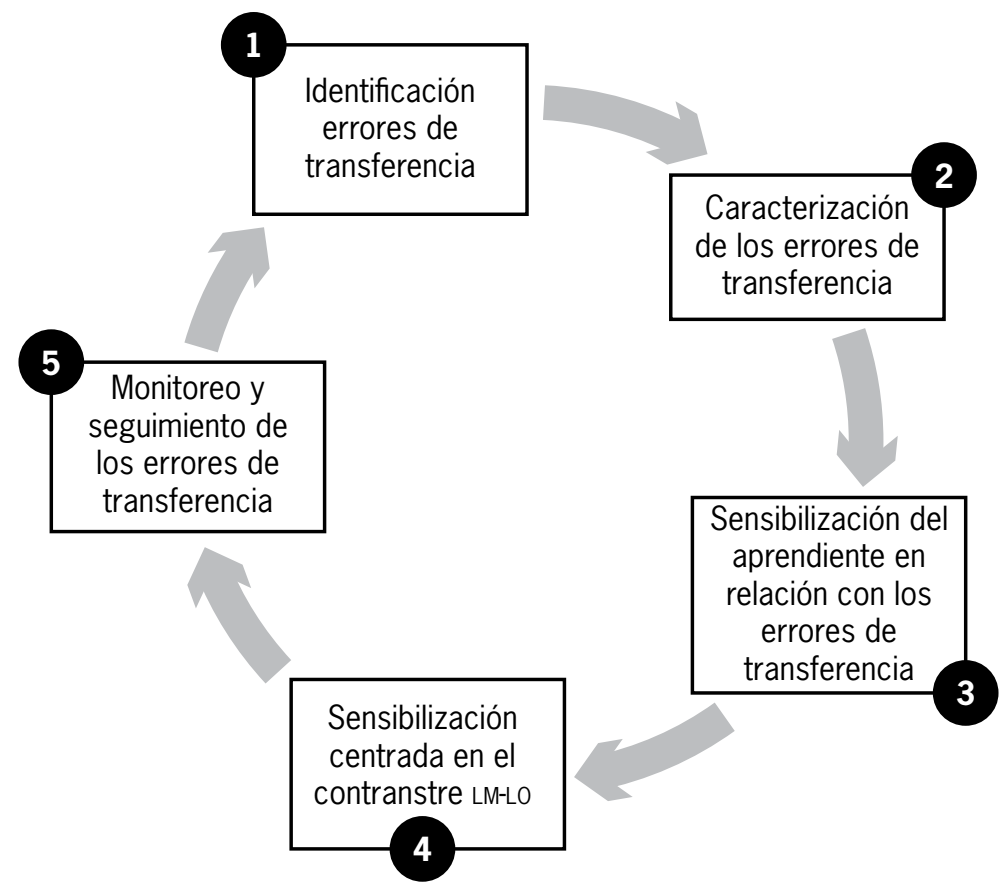

Figura 1. Modelo de tratamiento de errores de transferencia en la producción escrita en ambientes de aprendizaje tecnológicos

Fuente: elaboración propia.

de puntualizar los momentos en los que surge y el porqué de la interferencia según las diferencias o similitudes de la LM y la Lo por parte del estudiante. Este procedimiento es relevante, puesto que permite concienciar al estudiante en cuanto a la naturaleza del error para identificarlo con mayor precisión y realizar el respectivo seguimiento de este en su producción escrita. La caracterización como elemento clave en el proceso del tratamiento supone el reconocimiento que genera el estudiante en este tipo de errores y cómo puede monitorearlos para reparar su posible aparición.

Una vez terminado el proceso anterior, es necesario propiciar un espacio para la reflexión e indagación del estudiante sobre el error, la retroalimentación dirigida basada en el contraste entre la LM y la Lo y, finalmente, el monitoreo del error. En cada etapa, el profesor de lenguas o tutor de intercambio podría llevar al aprendiente a una mirada introspectiva de su lengua materna con el objeto de reconocer la influencia directa o indirecta que ejerce la LM sobre el aprendizaje de la Lo. A continuación, se explicita la forma de operacionalizarse las etapas para aplicar el modelo en una intervención correctiva.

\section{Etapa 1. Identificación de los errores de transferencia}

El modelo comienza con una de las etapas más importantes de todo proceso de intervención, esta es la identificación y delimitación de las formas lingüísticas a tratar. En este caso, para las comunidades de aprendizaje virtual, es necesario incluir en sus correcciones, la identificación de los errores a partir de los textos ya escritos de los aprendientes. Para ello, existen diversas metodologías, sin embargo, es necesario focalizar la problemática con la finalidad de tratar aquellos errores que, según el grado de interferencia o perturbación, afecten la comunicación o no evidencien una mayor precisión lingüística.

En relación con la metodología para la identificación de los errores acorde con los criterios establecidos por Corder (1967), tiene una implicancia distinta cuando se identifica errores de transferencia (Alexopoulou, 2005; Vázquez, 1991; Elejalde y 
Ferreira, 2016). Los errores de transferencia en sí tienen una complejidad contraria dada la caracterización del error proveniente de la lengua materna de los estudiantes. Aquí, el profesor o investigador requiere del conocimiento del sistema lingüístico de la lengua materna (a nivel operativo) ${ }^{4}$ para determinar si los errores identificados corresponden a la omisión, adición o falsa selección (sustitución o falsa colocación) de elementos provenientes de la lengua materna del aprendiente, si hay interferencias de otras lenguas aprendidas o si es producto de la internalización de las reglas gramaticales, pragmáticas o culturales de la LO (errores intralingüísticos). La identificación también dependerá del tipo de errores que el profesor busque tratar considerando la gravedad del error, si es global o local, si dificulta la comunicación o si es un error que podría fosilizarse. Sobre estas consideraciones, el profesor o investigador podría seguir los criterios que se muestran en la figura 2.

Como se observa en la figura 2, se propone un procedimiento basado en criterios del AE y la propuesta metodológica desarrollada por Ferreira y Elejalde (2017) en su modelo metodológico para análisis de frecuencia y recurrencia de errores. Esto con el fin de determinar los errores que serán tratados acorde con la clasificación (lingüísticos, léxicos o discursivos) y la sistematicidad de estos.

a. Análisis de textos ya escritos en la comunidad. Para ello, se sugiere realizar un primer acercamiento a la producción escrita del aprendiente seleccionando la producción de un texto escrito en la comunidad virtual o medio tecnológico que se pretende intervenir. La mayoría de estudiantes ha publicado al menos 5 textos a lo largo de un periodo no mayor a 3 meses, de este modo, convendría analizar e identificar los errores en el texto 1 . Se sugiere además que los textos tengan una extensión mínima por nivel, es decir, los de nivel A2-200 palabras, B1-300, C1-500 aproximadamente. En relación con el tipo de escrito, es necesario determinar si será expositivo, argumentativo o narrativo y la temática, puesto que cada estilo produce diferentes estructuras. Se sugiere, además, como procedimiento metodológico, identificar el texto escrito con mayor antigüedad (primer texto escrito o un texto escrito más allá de la fecha actual en la revisión) para considerarlo como fuente principal de focalización para los errores a tratar. Tomar como base estos textos de diferentes

CRITERIOS DE INVESTIGACIÓN DE ERRORES DE TRANSFERENCIA

Anállisis de textos ya escritos en la comunidad.
Fuente de escritura para determinar los errores a tratar más frecuentes.

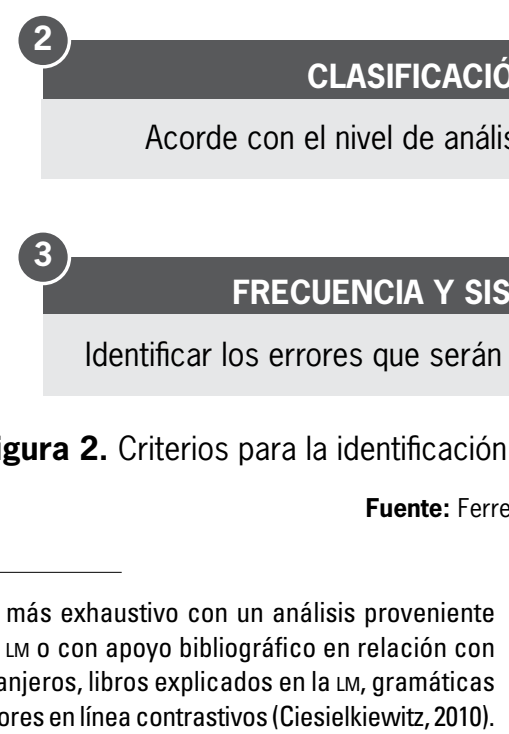

4 Se sugiere un apoyo más exhaustivo con un análisis proveniente de un traductor de la LM o con apoyo bibliográfico en relación con gramáticas para extranjeros, libros explicados en la LM, gramáticas contrastivas y traductores en línea contrastivos (Ciesielkiewitz, 2010). 
sujetos, permitirá al profesor o investigador establecer los errores más frecuentes y sistemáticos para orientar la intervención lingüística.

b. Clasificación del error. Los errores en sí se delimitan al sistema lingüístico de la Lo, sin embargo, considerar el origen del error supone una clasificación del sistema lingüístico acorde con los criterios de transferencia directa negativa. Esta clasificación corresponde a los distintos niveles en los cuales podrá jerarquizarse el error para un análisis posterior de los resultados recolectados. En este aspecto, se considera principalmente las clasificaciones a partir del sistema lingüístico (errores lingüísticos: gramaticales, sintácticos y morfológicos explicados a partir de la influencia de la lengua materna), errores léxicos, comunicativos, pragmáticos y culturales. Estas clasificaciones van seguidas de la descripción del error (taxonomía de su descripción) para caracterizarlo con criterios de omisión, adición, falsa selección, falsa colocación, forma errónea y sustitución (Vázquez, 1991; Alexopoulou, 2005; Ferreira, Elejalde y Vine, 2014; Elejalde y Ferreira 2016; Ferreira y Elejalde 2017). La clasificación de transferencia se plantea en la tabla 1.

Como se observa en la tabla 1, la trasferencia puede ocurrir en estructuras tanto gramaticales (omisión de preposiciones por interferencia de la LM, omisión del artículo, entre otros), como en otras instancias presentadas como cambio de código, calco, interferencia L2, entre otras. Esta clasificación permite reunir los datos en categorías que delimiten los errores en estudio acorde con los grados de interferencia, sean estos en la estructura gramatical, la estructura sintáctica, léxica, morfológica o discursiva. Con este criterio es posible categorizar y ordenar la jerarquización de los errores identificados y permitir la toma de decisiones para un tratamiento efectivo. Otro criterio importante será considerar el nivel de proficiencia del aprendiente, dado que los errores reflejan etapas de influencia tanto interlingüística como intralingüística en el desarrollo de la interlengua.

A partir de la clasificación de los errores, el profesor o investigador podrá determinar frecuencias y sistematicidad de las problemáticas más importantes sobre las cuales se debe aplicar un tratamiento efectivo.

c. Delimitación de la sistematicidad de los errores frecuentes. Una vez identificados los errores y clasificados, se procede a clasificar aquellos que permanecen en el tiempo y que deben intervenirse para su reparación. Este método propuesto por Ferreira (2017) en su estudio de análisis de errores para focalizar el tratamiento de errores en ELE, permite con mayor precisión centrarse en aquellos errores que se sostienen en el tiempo y podrían llegar a fosilizarse (tabla 2). Para este proceso, se sugiere hacer un conteo de los errores por cada aprendiente y cada texto escrito versus el error identificado frecuente

Tabla 1. Taxonomía para identificar errores de transferencia en los distintos niveles de la lengua

\begin{tabular}{|c|c|c|c|}
\hline Categoría & \multicolumn{2}{|c|}{ Tipo de error } & Descripción del error \\
\hline \multirow{11}{*}{ Interlinguales } & \multirow{6}{*}{ Transferencia } & Estructura gramatical & Adición (ADI) \\
\hline & & Estructura sintáctica & Omisión (омı) \\
\hline & & Léxica & Falsa selección (Fs) \\
\hline & & Morfología & Falsa colocación (FC) \\
\hline & & Gramática ortográfica & Forma errónea (FE) \\
\hline & & Discursiva & \\
\hline & \multicolumn{3}{|l|}{ Traducción literal } \\
\hline & \multicolumn{3}{|l|}{ Cambio de código } \\
\hline & \multicolumn{3}{|l|}{ Interferencia L2 } \\
\hline & \multicolumn{3}{|l|}{ Calco } \\
\hline & \multicolumn{3}{|l|}{ Falso cognado } \\
\hline
\end{tabular}

Fuente: Elejalde y Ferreira (2016). 
(se establece con anterioridad los 5 o 10 errores más frecuentes previos a tratar). Para identificar la sistematicidad del error se sugiere que cada aprendiente escriba al menos 5 textos, incluidos uno diagnóstico de inicio y uno final. Se puede realizar la identificación de la sistematicidad del error como se detalla en la tabla 2.

Una vez realizada la tabla por textos, sujeto y error, puede determinarse qué error se mantiene en el tiempo para así crear las listas de errores de transferencia. Luego de este análisis, se sugiere establecer según el número de sistematicidad final de 5 a 10 tipos de errores para intervenir. Con estos se establece el número de ejemplos contextualizados para contrastar y así apoyar la posterior intervención.

\section{Etapa 2. La caracterización del error de transferencia}

La caracterización es una de las fases más relevantes, dado que permite establecer un acercamiento entre el estudiante y los errores que son producto de la lengua materna. Esta etapa se operacionaliza en el momento de la retroalimentación del texto solicitado con la finalidad de presentar la problemática en cuestión. La mayoría de los aprendientes desconoce su sistema lingüístico por la automatización que tiene la LM en cada persona. Esto genera procesos mecánicos y automáticos de los que no son conscientes y, como consecuencia, en el proceso de aprendizaje de una LO, convendría una concienciación respecto del sistema lingüístico. En este sentido, los aprendientes no identifican autónomamente si los errores cometidos tienen su origen en la LM, situación que los sorprende cuando el profesor les ayuda a indagar si hay una interferencia en la producción escrita.

En el análisis de errores y de categorización, una vez establecido el acercamiento con el estudiante y su proceso de sensibilización (Alexopoulou, 2005; Vázquez, 1991; Fernández, 1991) se debe enfocar el tratamiento en la reflexión que debe llevar el profesor a la hora de intervenir los errores. Para ello, hay que caracterizar el error desde parámetros consensuados entre el aprendiente y el profesor que evidencien en próximas producciones errores de este tipo. Dichos parámetros permitirán la caracterización que determinará en qué medida la influencia de la LM o Lo interfiere en el aprendizaje y la comunicación en la Lo. Se sugiere establecer esta caracterización vía Skype o mensajería instantánea con el aprendiente.

La caracterización se propone según los siguientes parámetros:

a. Tipo de error (lingüístico, morfosintáctico, léxico, pragmático o cultural). En este primer aspecto, se declara qué tipo de error es, con el objeto de evidenciar en el aprendiente cuál es el nivel que afecta dentro de la comunicación. En esta instancia, es pertinente contar con listas de mensajes erróneos que faciliten al estudiante reflexionar si el error interfiere o no con la comunicación y la precisión lingüística en el mensaje.

Tabla 2. Ejemplo obtención de la sistematicidad del error

\begin{tabular}{|c|c|c|c|c|c|c|}
\hline \multirow{2}{*}{$\begin{array}{l}\text { Conteo por } \\
\text { estudiante }\end{array}$} & \multicolumn{4}{|c|}{ Tipo de error frecuente: omisión de la preposición "a" } & \multirow{2}{*}{ FR. Error } & \multirow{2}{*}{ Sistematicidad } \\
\hline & T1 & T2 & T3 & T4 & & \\
\hline Estudiante 1 & 2 & 2 & & 3 & 7 & 3 textos \\
\hline Estudiante 4 & 1 & 1 & 2 & & 4 & 3 textos \\
\hline \multicolumn{7}{|l|}{ Estudiante 20} \\
\hline & & & & & & \\
\hline & & & & & & \\
\hline Total estudiantes & & & & & Total FR & Total sist. \\
\hline
\end{tabular}

Fuente: Ferreira y Elejalde (2017). 
b. Descripción del error. En esta instancia, debe recurrirse al contraste entre las lenguas para ejemplificar adecuadamente las situaciones lingüísticas de la LM que podrían interferir en la producción de la Lo. Se sugiere la ejemplificación contrastiva del error versus la regla gramatical-léxica-morfosintácticapragmática, el contexto de uso en la LM y el uso en la LO.

c. Nivel de interferencia (nivel lingüístico, comunicativo, pragmático o cultural). Acorde con esta caracterización del error, el profesor de ELE podrá determinar si los errores más frecuentes caracterizados necesitan intervenirse según su gravedad. La frecuencia debería medirse de igual manera con un enfoque de análisis de datos sistemático como se sugirió anteriormente, cuya metodología plantea identificar si los errores son persistentes en el tiempo (Ferreira y Elejalde, 2017). Dicha persistencia indica si el error debe tratarse y no corresponde a una frecuencia cuyos resultados no reflejan el proceso de cada aprendiz evaluado. La caracterización del error ayudará al estudiante de ELE a internalizar el tipo de error proveniente de su LM.

En esta etapa de caracterización es importante tener en cuenta que solo forma parte de la descripción y acercamiento del aprendiente hacia los errores de transferencia. Por tanto, no debe existir o propiciarse en este paso una retroalimentación o corrección, que produzca un efecto contrario a la sensibilización del error.

\section{Etapa 3. La sensibilización y reflexión metalingüística sobre el error de transferencia}

En el tratamiento de los errores, tanto el profesor como el aprendiente se han enfocado en los errores de orden gramatical, léxico o sintáctico a partir de una explicación que atañe al sistema lingüístico de la Lo. Sin embargo, escasamente se ha dado prioridad a la posible causa $u$ origen de estos errores que, en este caso, pueden surgir por la influencia directa o indirecta da la LM. Asimismo, los errores de transferencia, como muchos otros de tipo comunicativo o pragmático, no son considerados relevantes dadas las características que ha posicionado al fenómeno de la transferencia en desventaja con otros procesos subyacentes de la interlengua. En este aspecto, es pertinente considerar que los errores de transferencia son precisamente aquellos que interfieren, como en el caso de esta investigación, de forma negativa afectando la fluidez y precisión lingüística en el mensaje acorde con la situación comunicativa en la Lo. De hecho, muchos de los errores de tipo gramatical tienen su explicación a partir del análisis de la lengua materna del aprendiz y del sistema lingüístico transferido en las reglas de la Lo. Por tanto, llevar al aprendiente a identificar cuáles de estos errores son producto de la LM, le ayudarán a reconocer la

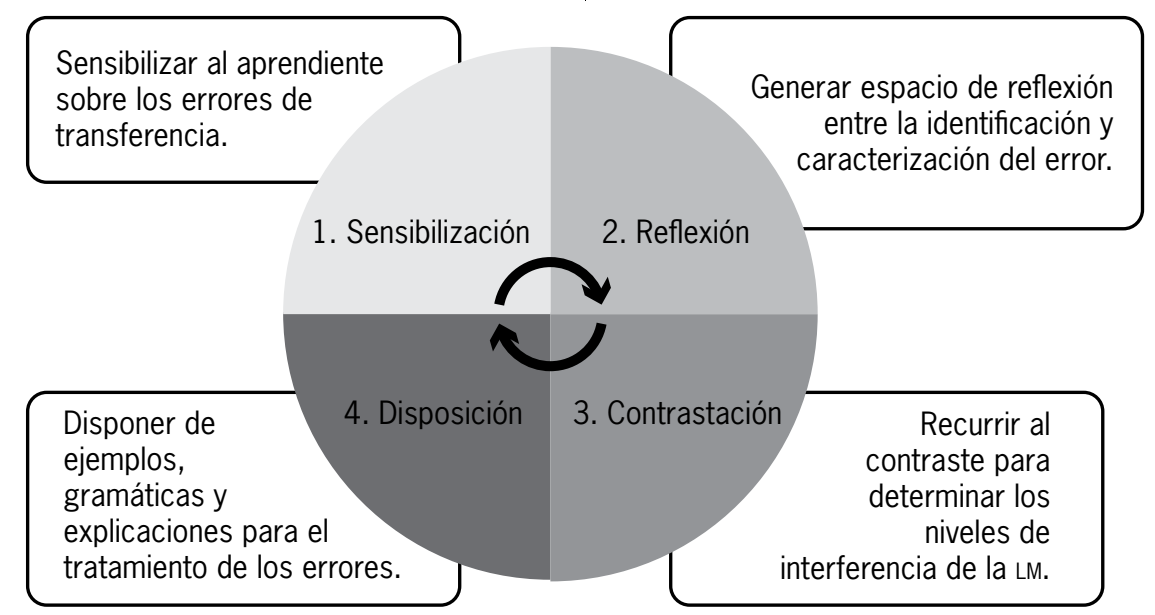

Figura 3. Proceso concienciación del error de transferencia en el aprendiente 
diferencia entre la L2 y su LM para reparar el error de forma eficaz. Esta concientización por parte del aprendiz puede desarrollarse como se explica en la figura 3.

a. La sensibilización. La figura 3 muestra un ciclo que comienza con la sensibilización del aprendiente frente a los errores que provienen de la LM. Este ciclo se repite cada vez que sea necesario para generar los espacios de sensibilización durante el tratamiento de los errores. Aquí, se sugiere preparar un cuestionario para el aprendiente como se propone a continuación:

- Sensibilizar al estudiante sobre los errores provenientes de la LM:

- ¿Crees que los errores de tu (LO) provienen de la (LM)?: ejemplo, ¿Crees que los errores de tu español provienen del inglés?

- ¿Hay alguna relación entre los errores de la (LO) identificados con la (LM)?

Una vez establecidas estas preguntas se dispone al aprendiente a reconocer los errores provenientes de la LM con el objeto de brindar el espacio para investigar el error por parte del aprendiz. Se sugiere la presentación de los errores como se ejemplifica en la figura 4.

Así como se establece en la identificación del error por parte de autores que indican el señalamiento del error (Corder, 1967; Vázquez, 1991; Fernández, 1991, 1997; Alexopolou, 2005), la figura 4 muestra una forma de evidenciar los errores identificados de la LM. Los asteriscos funcionan como indicadores de algo incorrecto, y los paréntesis corresponden a la omisión de un elemento en la oración.

Se sugiere entregar al estudiante una serie de ejemplos como el anterior para determinar cierto tipo de colocaciones o estructuras que puedan identificarse como provenientes de la LM del aprendiente.

b. Propiciar la reflexión del estudiante de ELE. Una vez completada la fase anterior, se debe propiciar al estudiante de ELE un espacio de reflexión por cada error a tratar donde se le sugiere la reparación del este. Por lo general, en la corrección lingüística se hace alusión principalmente a la parte gramatical o léxica correcta en la LO. Pocas veces se observa en los textos recolectados o corregidos información acerca de la influencia de la lengua materna y la diferencia entre la Lo.

Por estas razones, dentro del modelo de tratamiento, se sugiere considerar preguntas metalingüísticas que ayuden al estudiante concienciarse sobre la importancia de conocer su propio sistema lingüístico y reparar el error en su Lo. De este modo, el estudiante entra en contacto con la función metalingüística del lenguaje, permitiéndole reconocer cómo en su LM funciona determinada regla gramatical y cómo esta podría ser diferente en la Lo. Para lograr este tipo de reflexión, se sugieren las siguientes preguntas didácticas ${ }^{5}$ para conducir la reflexión del error de transferencia, cuya base podría partir de la construcción de una rúbrica con una escala de evaluación que considere estos ítems:

- ¿Dequémanera has utilizado tu lengua materna para solucionar este vacío de información?

- ¿Consideras que usaste tu lengua materna de forma automática o pensaste en la estructura antes de usarla?

- ¿Cuál sería la forma esperada en la lengua que estudias?

- ¿Cómo puedes corregirla en escritos futuros?

\section{- Me gusta $\underline{a}^{*}$ ayudar $(-\mathbf{a}-)^{*}$ mis amigos $(-\mathbf{a}-)^{*}$ practicar inglés.}

Figura 4. Ejemplificación del error del aprendiente

Fuente: elaboración propia.

5 Para estas preguntas se sugiere adecuar la estructura presentada en la figura 4 y el contenido acorde con el nivel de proficiencia y el nivel de comprensión de los estudiantes. 
Una vez se establece la reflexión, conviene desarrollar una lista de posibles explicaciones y usos de la LM que han ejercido influencia en la Lo del aprendiente. De esta manera, se logra mayor conciencia sobre las posibles causas que interfieren en la comunicación a la hora de suplir vacío de información en la LO.

c. Contrastación. Una vez establecida la reflexión, se procede a la siguiente etapa relacionada con la contrastación, dirigida en estructuras de la LM y la Lo. Luego del contacto entre el estudiante y sus errores, se debe guiar la percepción y reconocimiento con otro set de preguntas claves:

- De los siguientes errores, ¿cuáles de estos crees que se parecen a una estructura de tu lengua materna?

- ¿Cuáles provienen de la combinación entre tu lengua materna y la lengua que aprendes?

- ¿Cómo crees que afecta esto a la comunicación en la lengua que aprendes?

A partir de estas preguntas que responden a la identificación del error, se procede a la comparación contrastiva entre los errores identificados entre la LM y la LO, como se sugiere en la figura 5.

En la figura 5 se muestra un análisis de los errores que provienen de la LM. La diagramación contrastiva de estos permite al estudiante identificar los elementos transferidos que inducen a error. Así, en la figura 5 se observa cómo el aprendiente de L1 inglés transfirió la traducción literal de la preposición "to", la omisión de la preposición "a” para introducir complemento de persona y, finalmente, la omisión o no traspasó particularmente de la preposición "to"*.
Con esta diagramación del error, el estudiante de ELE podrá comparar si el error efectivamente proviene de su LM o si solo forma parte de su desarrollo. Esta diagramación se realiza a partir de los errores sistemáticos delimitados en la etapa anterior. En estudios sobre feedback correctivo, Contreras y Ferreira (2013), en su estudio en relación con los errores del subjuntivo en español, introdujeron la contrastación para guiar a los aprendientes a notar que la Lo no procede de la misma manera que en la LM.

d. Disposición. En este paso se sugiere contar con listados de errores de transferencia con sus respectivos análisis para identificar con mayor precisión aquellos errores que corresponden a ciertas estructuras de la LM. La disposición de este material permitirá avanzar al estudiante hacia la sensibilización dirigida en el contraste LM-LO y, como consecuencia, al monitoreo de su aprendizaje.

\section{Etapa 4. Sensibilización dirigida en el contraste LM-LO}

Como ya se ha resaltado, crear una conciencia sobre el error de transferencia facilitará establecer en el estudiante una ruta de identificación para así reparar el error. Este derrotero, como se ha expuesto a lo largo de las etapas mencionadas, confluye en la retroalimentación dirigida por el docente de ELE a partir del contraste entre la LM y la Lo. Este hará que el estudiante repare el error considerando las reglas de la LO y la LM. En casos en los que se favorezca la transferencia, también se debería realzar la importancia de identificar la interferencia positiva que ejerce la LM. Asimismo, se sugiere en esta etapa contar con diccionarios gramaticales, análisis

\section{- Me gusta $\underline{a}^{*}$ ayudar (_a_)* mis amigos (_a_)* practicar inglés. $\checkmark$ $\checkmark$ - I like to help (_0_)* my friends to ${ }^{*}$ practice english}

Figura 5. Análisis comparativo y contrastivo de los errores a intervenir 
provenientes de otros profesores de inglés o de traductores de las lenguas involucradas. Dichos análisis enriquecerán la información obtenida de la LM del aprendiz, la cual debe usarse para la reparación del error. Para lograr esta etapa, se sugiere que el aprendiente junto con el profesor o investigador, estructuren las relaciones contrastivas de los errores identificados como se sugirió en la figura 5. Así se crea un elemento de identificación del aprendiente para reconocer y caracterizar los errores que interfieren producto de su LM.

\section{Etapa 5. Monitoreo y seguimiento de los errores}

Consiste en introducir al aprendiente en intervenciones lingüísticas diseñadas por el profesor para iniciar una reparación a lo largo del tiempo de los errores identificados. Para ello, se sugiere que, acorde con el modelo de intervención, el estudiante monitoree la aparición de este tipo de errores cuando se realicen los textos escritos o actividades de escritura. En este sentido, las correcciones que haga el docente podrán concretarse si el estudiante hace seguimiento a la identificación de los errores de transferencia y sensibilizándose para tener mayor agudeza en su escritura. Esto contribuirá a una mayor conciencia sobre la precisión gramatical considerando que existen elementos estructurales de la Lm que afectan el sistema lingüístico de la LO.

El monitoreo podrá adelantarse por medio de una matriz generada por el profesor para registrar el número de veces de apariciones, el tipo de error y su explicación. Finalizada la intervención, el aprendiente junto con el profesor podrán cotejar la evolución y el grado de sensibilización que tiene aquel con respecto a las interferencias provenientes de la LM. En esta última etapa, además, se sugiere implementar estrategias de feedback correctivo propuestas por Contreras y Ferreira (2013), donde la transferencia cobra importancia a la hora de tratar problemáticas en la LO.

\section{Conclusiones}

Numerosos investigadores en el ámbito de la ASL a menudo han planteado en sus conclusiones dudas sobre la efectividad de la aplicación de los resultados de una investigación a la enseñanza de lenguas. Hatch (1978), desde sus inicios, reconoció la necesidad de cautelar la disposición de metodologías basadas en resultados de una investigación, puesto que los escenarios de aprendizaje y los aprendientes están sujetos a variables diferentes y cambios constantes. Otros autores (Lightbown, 2000) sugieren en esta misma línea que los resultados de investigaciones pueden ser de escaso valor para la formación de profesores; debido a que el aula y los ambientes de aprendizaje son dinámicos y las generaciones de aprendientes cada vez son más digitalizadas e inmersas en un mundo donde la tecnología cobra especial valor. Así, los resultados de una investigación, permiten, en palabras de Lightbown (2000), educar a los profesionales en el área de enseñanza de lenguas, para entender qué se puede o no lograr en el aula de lenguas. En consecuencia "las investigaciones en ASL no constituyen la única -ni la principal-fuente de información para guiar a los profesores en su práctica diaria" (Lightbown, 2000, p. 454). No obstante, en el caso de la investigación, los modelos de análisis y procesamiento de los errores en estudio, permiten dilucidar un procedimiento y metodología que apoye el tratamiento de errores producto de la LM, los cuales han sido abordados, pero con escasa información sobre cómo tratarlos.

De este modo, la propuesta de un modelo de tratamiento pone en relieve la importancia de tratar los errores de transferencia como parte del aprendizaje de una L2. En la mayoría de veces, la reparación y corrección del error tienen un enfoque más gramatical y léxico fuera de todo contexto que conduzca al aprendiente a identificar si hay una influencia directa o no de su lengua materna. Tanto en el aula de clases como en ambientes virtuales de aprendizaje, la estrategia común es corregir el error centrado en la explicación gramatical, el uso en la Lo y como este error afecta a la comunicación, relegando así la importancia de considerar la LM u otras lenguas aprendidas.

De lo anterior puede deducirse que el objetivo de un análisis en la investigación existente, más los estudios conducentes a obtener mayor información 
sobre los procesos que subyacen a la conformación de la interlengua, no es prescribir o indicar lo que los profesores deben realizar en el aula de clases para garantizar una enseñanza y aprendizaje efectivos. Más bien, consiste en propiciar espacios de reflexión sobre la complejidad que en sí caracteriza el aprendizaje, más cuando existen fenómenos de la lengua que interfieren en la Lo por el sistema neurolingüístico que posee el ser humano (MacWhinney, 2002). Asimismo, se sugiere, con la investigación y los modelos, estimular la disposición de los profesores e investigadores a experimentar con nuevos planteamientos y enfoques según las condiciones particulares del ambiente de aprendizaje, las características individuales de los aprendientes y las estructuras a tratar.

Por consiguiente, el modelo de tratamiento expuesto responde a una de las necesidades de abordar principalmente los errores producto de la influencia de la LM, dado que la mayoría de veces los docentes de ELE se centran en corregir y reparar el error, desconociendo la procedencia y la explicación de este. En este sentido, podría señalarse, sin ser concluyente, que la metodología ha sido intuitiva y enfocada en reparar el error léxico-gramatical por parte del docente sin intervenir específicamente en áreas como la transferencia para solucionar aquellos errores reiterativos. Con la propuesta del modelo, se espera contribuir a un procedimiento en materia de identificación, caracterización y tratamiento del error de transferencia, siguiendo los conductos de sensibilización, categorización y tratamiento de errores propuestos por diversos autores en materia de reparación de estos. En este modelo se releva la importancia de concienciar al estudiante de ELE en relación con sus falencias y errores durante la situación comunicativa que corresponda, así como lo expone Alexopoulou (2005) en su estudio para el tratamiento de errores desde el punto de vista del aprendiente.

Asimismo, dicho modelo, al ser una versión no concluyente e incipiente en su propuesta metodológica, conviene realizar futuras investigaciones en las que se pruebe el modelo para determinar la efectividad en la corrección de los errores, y si su implementación permite elegir con mayor acierto las estrategias de feedback correctivo. En estas últimas, cabe destacar la necesidad de incluir propuestas de feedback relacionadas con los fenómenos estudiados, como en el caso de la transferencia, propuesto por Contreras y Ferreira (2013), donde las estrategias de recast y metalingüísticas, se apoyan en el conocimiento y reflexión de la lengua materna.

Por último, el modelo trabajado en esta investigación es de tipo sugerente y está sujeto a modificaciones con respecto a su utilización en cualquier ambiente de aprendizaje, ajustándose así a las necesidades, intereses y objetivos tanto del investigador como del aprendiente de lenguas.

En la misma línea de Alexopoulou (2005), Vázquez $(1991,2009)$ y Contreras y Ferreira (2013), el tratamiento de errores debe ser un recurso didáctico destinado a favorecer el aprendizaje y automatización de las habilidades en una LO. El profesor de lenguas debe guiar a sus aprendientes en el tratamiento, en procura de adecuar el modelo de intervención correctiva a los objetivos, métodos y necesidades particulares. De este modo, para que la práctica correctiva sea efectiva, el profesor debe fomentar la autonomía y el monitoreo del aprendiente, haciéndolo partícipe y responsable en el complejo proceso de tratamiento de errores de la LO. Así, con el apoyo de estrategias de feedback y modelos de tratamiento, la reestructuración de la interlengua y la reconfiguración de las hipótesis falsas pueden resultar en el mejoramiento de las habilidades lingüísticas en la LO.

\section{Referencias}

Alexopoulou, A. (2005). Aproximación al tratamiento del error en la clase de ELE desde la perspectiva del análisis de errores. Estudios de Lingüística Aplicada, 23(41), 101-125.

Alexopoulou, A. (2006). Los criterios descriptivo y etiológico en la clasificación de los errores del hablante no nativo: Una nueva perspectiva. Porta Linguarum, $5,17-35$. 
Alexopoulou, A. (2011). El papel de la transferencia en los errores léxicos. Revista Nebrija de Lingüística Aplicada, 9(5), 27-36.

Cassany, D. (2006). Análisis de una práctica letrada electrónica. Páginas de Guarda, (2), 99-112.

Ciesielkiewicz, M. (2010). El resurgir de la lingüística contrastiva en los manuales de lengua polaca. Eslavística Complutense, 10, 83-95.

Corder, S.P. (1967). The significance of learner's errors. International Review of Applied Linguistics in Language Teaching, 5, 1-4.

Contreras, C. y Ferreira, A. (2013). Orden de adquisición en español como lengua extranjera: modalidad subjuntiva. Literatura y Lingüística, 28, 249-283.

Elejalde, J. y Ferreira, A. (2016). Errores de transferencia en comunidades de aprendizaje en línea por aprendientes de español como lengua extranjera (ele). Trabalhos en Lingüística, 55(3), 619-650.

Fernández, S. (1991). Interlengua y análisis de errores en el aprendizaje del español como lengua extranjera. (Tesis doctoral). Universidad Complutense de Madrid, Madrid.

Fernández, S. (1997). Interlengua y análisis de errores: en el aprendizaje del español como lengua extranjera. Madrid: Edelsa Grupo Didascalia.

Ferreira, A. (2006). Estrategias efectivas de feedback positivo y correctivo en el español como lengua extranjera. Revista Signos, 39(62), 379-406.

Ferreira, A. (2017). El efecto del feedback correctivo para mejorar la destreza escrita en ELE. Colombian Applied Linguistics Journal, 19(1), 37-50.

Ferreira, A y Elejalde, J. (2017). Análisis de errores recurrentes en un corpus de aprendices de español como lengua extranjera (Corpus CAELE). Revista Brasileira de Linguística Aplicada, 17(3), 509-538.

Ferreira, A. y Kotz, G. (2010). ELE-TUTOR Inteligente: un analizador computacional para el tratamiento de errores gramaticales en español como lengua extranjera. Revista Signos. Estudios de Lingüística, 43(73), 211-236.

Ferreira, A. y Lafleur, N. (2015). Analyse et description des erreurs prépositionnelles les plus fréquentes en espagnol L2. Lingüística y Literatura, 68, 57-79.
Ferreira, A., Elejalde, J. y Vine, A. (2014). Análisis de errores asistido por computador basado en un corpus de aprendientes de español como lengua extranjera. Revista Signos, 47(86), 385-411.

Ferreira, A., Salcedo, P., Kotz, G. y Barrientos, F. (2012). La arquitectura de ELE-TUTOR: un sistema tutorial inteligente para el español como lengua extranjera. Revista Signos, 45(79), 102-131.

Gargallo, I.S. (1994). Análisis de errores: valoración gramatical y comunicativa en la expresión escrita de estudiantes de ELE. Una propuesta didáctica. En Actas del Segundo Congreso Nacional de ASELE: español para extranjeros: didáctica e investigación (pp. 169-174). Madrid, del 3 al 5 de diciembre de 1990.

Hatch, E. (1978). Discourse analysis and second language acquisition. En E. Hatch (ed.), Second language acquisition (pp. 383-400). Rowley, Mass.: Newbury House

Lamy, M. y Hampel, R. (2007). Online communication in language teaching and learning. Australia: Palgrave Macmillan.

Lightbown, P. (2000). Anniversary article. Classroom SLA research and second language teaching. Applied Linguistics, 21(4), 431-462.

Long, M. H., The role of the linguistic environment in second language acquisition. En W.C. Ritchie y T.K. Bhatia (eds.), Handbook of second language acquisition (Vol. 2: Second language acquisition, pp. 413-468). Nueva York: Academic.

MacWhinney, B. (2002). Extending the competition model. Advances in Psychology, 134, 31-57.

Maldonado, R. (2013). Análisis de errores en la adquisición/aprendizaje del español en estudiantes letones. (Tesis de magíster). Universidad de Alcalá, Madrid.

Vázquez, G.E. (1991). Análisis de errores y aprendizaje de español/lengua extranjera. Nueva York: Peter Lang Pub.

Vázquez, G. (2009). El concepto de error: estado de la cuestión y posibles investigaciones. Revista Nebrija de Lingüística Aplicada a la Enseñanza de Lenguas, Revista Nebrija de Lingüística Aplicada, 5(3), 104-114.

Warschauer, M. (2001). Online communication. En R. Carter y D. Nunan (eds.), The Cambridge guide to teaching English to speakers of other languages (pp. 207212). Cambridge: Cambridge University Press.

\section{Para citar este artículo}

Elejalde Gómez, J. y Ferreira Cabrera, A. (2018). Modelo para un tratamiento de errores de transferencia en la escritura en ELE producida en comunidades virtuales.

Folios, 48, 137-152. 